\title{
CORRIGENDUM TO NEW INEQUALITIES ON HOMOGENEOUS FUNCTIONS, J. INDONES. MATH. SOC. 15 (2009), NO. 1, 49-59
}

\author{
V. LOKesha ${ }^{1}$, K.M. NAgArajA ${ }^{2}$, And Y. SimseK ${ }^{3}$ \\ ${ }^{1}$ Department of Mathematics, Acharya Institute of Technology, \\ Soldevana- halli, Bangolore-90, India \\ lokiv@yahoo.com \\ ${ }^{2}$ Department of Mathematics, Sri Krishna Institute of Technology,
} Chikkabanavara, Hesaraghata Main Road, Karnataka, Bangolore-90, India 2406@yahoo.co.in

${ }^{3}$ University of Akdeniz, Faculty of Arts and Science, Department of Mathematics, 07058, Antalya, Turkey ysimsek@akdeniz.edu.tr

The paper contains typing errors.

Theorem 2 Let $\mu_{1}, \mu_{2} \in(-2, \infty), r<1$. If $\mu_{1} \leq \frac{4}{1-r} \leq \mu_{2}$, then

$$
g n_{\mu_{2}, r}(a, b) \leq L(a, b) \leq G n_{\mu_{1}, r}(a, b) .
$$

Furthermore $\mu_{1}=\mu_{2}=\frac{4}{1-r}$ is the best possibility for inequality (12). Also for $r=0$,

$$
g n_{\mu_{2}, 0}(a, b) \leq L(a, b) \leq G n_{\mu_{1}, 0}(a, b) .
$$

Furthermore $\mu_{1}=\mu_{2}=4$ is the best possibility for inequality (13).

Theorem 3 For $\mu_{1}, \mu_{2} \in(-2, \infty), r \neq \frac{2}{3}, r<1$ and if $\mu_{1} \leq \frac{2}{2-3 r} \leq \mu_{2}$, then

$$
g n_{\mu_{2}, r}(a, b) \leq I(a, b) \leq G n_{\mu_{1}, r}(a, b) .
$$

Furthermore $\mu_{1}=\mu_{2}=\frac{2}{2-3 r}$ is the best possibility for inequality (15). Also for $r=0$,

$$
g n_{\mu_{2}, 0}(a, b) \leq I(a, b) \leq G n_{\mu_{1}, 0}(a, b) .
$$

Furthermore $\mu_{1}=\mu_{2}=1$ is the best possibility for inequality (16).

Theorem 4 For $\mu_{1}, \mu_{2} \in(-2, \infty), r \neq 0$ and if $\mu_{2} \leq \frac{2}{r}-2 \leq \mu_{1}$, then

$$
g n_{\mu_{2}, 0}(a, b) \leq M_{r}(a, b) \leq G n_{\mu_{1}, 0}(a, b) .
$$

Furthermore $\mu_{1}=\mu_{2}=\frac{2}{r}-2$ is the best possibility for inequality (17) 
The above Theorems should be corrected to as follows:

Theorem 2 For $r \neq \frac{1}{3}$ and $\mu_{1}, \mu_{2} \in(-2, \infty)$ such that $\mu_{1} \leq \frac{4}{1-3 r} \leq \mu_{2}$, then

$$
g n_{\mu_{2}, r}(a, b) \leq L(a, b) \leq G n_{\mu_{1}, r}(a, b) .
$$

Furthermore $\mu_{1}=\mu_{2}=\frac{4}{1-3 r}$ is the best possibility for inequality (12). Also for $r=0$,

$$
g n_{\mu_{2}, 0}(a, b) \leq L(a, b) \leq G n_{\mu_{1}, 0}(a, b) .
$$

Furthermore $\mu_{1}=\mu_{2}=4$ is the best possibility for inequality (13).

Theorem 3 For $r \neq \frac{2}{3}$ and $\mu_{1}, \mu_{2} \in(-2, \infty)$ such that $\mu_{1} \leq \frac{2}{2-3 r} \leq \mu_{2}$, then

$$
g n_{\mu_{2}, r}(a, b) \leq I(a, b) \leq G n_{\mu_{1}, r}(a, b) .
$$

Furthermore $\mu_{1}=\mu_{2}=\frac{2}{2-3 r}$ is the best possibility for inequality (15). Also for $r=0$,

$$
g n_{\mu_{2}, 0}(a, b) \leq I(a, b) \leq G n_{\mu_{1}, 0}(a, b) .
$$

Furthermore $\mu_{1}=\mu_{2}=1$ is the best possibility for inequality (16).

Theorem 4 For $r \neq 1$ and $\mu_{1}, \mu_{2} \in(-2, \infty)$ such that $\mu_{2} \leq \frac{r}{1-r} \leq \mu_{1}$, then

$$
g n_{\mu_{2}, 0}(a, b) \leq M_{r}(a, b) \leq G n_{\mu_{1}, 0}(a, b) .
$$

Furthermore $\mu_{1}=\mu_{2}=\frac{r}{1-r}$ is the best possibility for inequality (17).

Remark. Carlson [1] and Lin [2] gave some inequalities on mean and logarithmic mean.

\section{REFERENCES}

[1] Carlson, B.C., "The logarithmic mean", Amer. Math. Monthly, 79 (1972), 615-618.

[2] Lin, T.P., "Mean and logarithmic mean", Amer. Math. Monthly, 81 (1974), 879-883. 